\title{
UTILIZAÇÃO DE DIFERENTES VOLUMES DE SÊMEN NA REALIZAÇÃO DO TESTE HIPOSMÓTICO EM TOUROS ADULTOS DA RAÇA NELORE
}

Leonardo Franco Martins ${ }^{1}$

Rogério Oliveira Pinho² José Domingos Guimarães ${ }^{3}$ Gustavo Guerino Macedo ${ }^{4}$ André Giarola Boscarato Douglas Potratz Rodrigues ${ }^{6}$ Silvio Henrique Dias ${ }^{7}$

MARTINS, L. F.; PINHO, R. O.; GUIMARÃES, J. D.; MACEDO, G. G.; BOSCARATO, A. G.; RODRIGUES, D. P.; DIAS, S. H. Utilização de diferentes volumes de sêmen na realização do teste hiposmótico em touros adultos da raça Nelore. Arq. Ciênc. Vet. Zool. UNIPAR, Umuarama, v.18, n.1, p. 5-10, jan./mar. 2015.

RESUMO: O objetivo deste estudo foi avaliar diferentes proporções de sêmen: solução hiposmótica na realização do teste hiposmótico e suas relações com a congelabilidade do sêmen de touros zebuínos. Utilizaram-se 15 ejaculados de três touros adultos da raça Nelore. No sêmen in natura realizou-se a avaliação física e morfológica, a coloração supravital e o teste hiposmótico. No teste hiposmótico foi utilizada uma solução com osmolaridade de $100 \mathrm{mOsm} / \mathrm{Kg}$ com 15 minutos de período de incubação a $37^{\circ} \mathrm{C}$, tanto no sêmen in natura quanto no congelado/descongelado. Foram utilizados quatro volumes de sêmen em $1 \mathrm{~mL}$ de solução hiposmótica: 10, 20, 50 e $100 \mu \mathrm{L}$. As amostras criopreservadas foram descongeladas e foram realizados os testes hiposmótico, coloração supravital, teste de termo-resistência lento e a coloração fluorescente. Os valores médios e desvios padrão do percentual de espermatozoides reativos ao teste hiposmótico em sêmen in natura e congelado/ descongelado foram $69,3 \pm 11,8$ e 20,5 $\pm 6,8$; respectivamente. Não houve correlação do teste hiposmótico com os aspectos físicos e morfológicos e os testes complementares realizados em sêmen in natura e congelado/descongelado. Nenhum teste de integridade de membrana plasmática dos espermatozoides foi capaz de classificar os touros quanto a sua congelabilidade do sêmen. Conclui-se que o teste hiposmótico pode ser realizado com 20 a $100 \mu \mathrm{L}$ de sêmen in natura, e 10 a $100 \mu \mathrm{L}$ de sêmen congelado/descongelado em $1 \mathrm{~mL}$ de solução hiposmótica, sem interferir em seus resultados, mas deve-se optar por 100 $\mu \mathrm{L}$ tanto para sêmen in natura e congelado/descongelado, porque melhora consideravelmente a leitura das lâminas.

PALAVRAS-CHAVE: Bos indicus. Criopreservação. Macho. Testes Complementares.

\section{USE OF DIFFERENT SEMEN VOLUMES IN HYPOOSMOTIC TEST OF ADULT NELORE BULLS}

ABSTRACT: The objective of this study was to evaluate different proportions of semen: hypoosmotic solution in the hypoosmotic swelling test and their relationship with semen freezability in Zebu bulls. A total of 15 ejaculates from three adult Nelore bulls were used. Physical and morphological features were analyzed in fresh semen, as well as supravital staining and hypoosmotic swelling test. In the hypoosmotic test, a hypoosmotic solution with $100 \mathrm{mOsm} / \mathrm{kg}$ osmolality using 15 minutes incubation at $37^{\circ} \mathrm{C}$ was used both in fresh and frozen/thawed semen. Four semen volumes in 1-ml hyposmotic solution were used: 10, 20, 50 and $100 \mu \mathrm{L}$. Cryopreserved samples were thawed and submitted to hypoosmotic tests, supravital staining, slow thermo-resistance test and fluorescent staining. Mean values and standard deviations of the percentage of reactive sperm cells in the hypoosmotic test in fresh and frozen/thawed semen were $69.3 \pm 11.8$ and $20.5 \pm 6.8$, respectively. There was no correlation between the hypoosmotic test and physical and morphological features and the complementary tests performed on fresh and frozen/thawed semen. None of the plasma membrane integrity tests was able to predict bull semen freezability. It can be concluded that the hypoosmotic test can be performed with 20 to $100 \mu \mathrm{L}$ fresh semen, and 10 to $100 \mu \mathrm{L}$ of frozen/ thawed semen in $1 \mathrm{~mL}$ of hypoosmotic solution without interfering with their results, but $100 \mu \mathrm{L}$ should be used in both, because it considerably improves the view of the slides.

KEYWORDS: Bos indicus. Cryopreservation. Male. Complementary test.

\section{EL USO DE DIFERENTES VOLÚMENES DE SEMEN EN EL TEST HIPOSMÓTICO EN TOROS ADULTOS DE LA RAZA NELORE}

RESUMEN: El objetivo de este estudio ha sido evaluar diferentes proporciones de semen: solución hiposmótica en la re-

DOI: https://doi.org/10.25110/arqvet.v18i1.2015.5362

'Professor Titular do curso de Medicina Veterinária e do Programa de Mestrado em Ciência Animal - Universidade Paranaense - UNIPAR. leonardofmpr@ yahoo.com.br;

${ }^{2}$ Pós doutorando no curso de Zootecnia da Universidade Federal de Viçosa;

${ }^{3}$ Professor Titular do curso de Medicina Veterinária da Universidade Federal de Viçosa;

${ }^{4}$ Professor Titular do curso de Medicina Veterinária da Universidade Federal de Uberlândia;

${ }^{5}$ Mestre pelo Programa de Mestrado em Ciência Animal - Universidade Paranaense - UNIPAR

${ }^{6}$ Mestrando pelo Programa de Mestrado em Ciência Animal - Universidade Paranaense - UNIPAR

${ }^{7}$ Acadêmico do curso de Medicina Veterinária, bolsista PIBIC-UNIPAR. 
alización del test hiposmótico y sus relaciones con la congelabilidad del semen de toros cebú. Se utilizaron 15 eyaculados de tres toros adultos de la raza Nelore. En el semen fresco se realizó evaluación física y morfológica, la tinción supravital y test hiposmótico. En el test hiposmótico se ha utilizado una solución con osmolaridad de $100 \mathrm{mOsm} / \mathrm{kg}$ con un período de incubación de 15 minutos a $37^{\circ} \mathrm{C}$, tanto en el semen fresco cuanto en el congelado/descongelado. Fueron utilizados cuatro volúmenes de $1 \mathrm{~mL}$ de solución hiposmótica: 10, 20, 50, y $100 \mu \mathrm{L}$. Las muestras criopreservadas fueron descongeladas y realizados los tests hiposmótico, tinción supravital, test de resistencia al fuego lento y la tinción fluorescente. Los valores medios y desvío estándar del porcentaje de espermatozoides reactivos al test hiposmótico en 1 semen fresco y congelado/descongelado fueron $69,3 \pm 11,8$ y 20,5 $\pm 6,8$; respectivamente. No hubo correlación del test hiposmótico con las características físicas y morfológicas y pruebas adicionales en el semen fresco y congelado/descongelado. Ningún test de integridad de la membrana plasmática de los espermatozoides han sido capaz de clasificar a los toros cuanto su congelabilidad del semen. Se puede concluir que el test hiposmótico puede ser realizado con 20 a $100 \mu \mathrm{L}$ de semen fresco, y de 10 a $100 \mu \mathrm{L}$ de semen congelado/descongelado en $1 \mathrm{~mL}$ de solución hiposmótica, sin interferir en sus resultados, pero se debe optar por $100 \mu \mathrm{L}$ para el semen fresco y congelado/descongelado, porque mejora significativamente la lectura de las láminas.

PALABRAS CLAVE: Bos indicus. Criopreservación. Macho. Testes complementares.

\section{Introdução}

A integridade funcional da membrana plasmática dos espermatozoides deve ser utilizada como rotina na avaliação de sêmen congelado/descongelado, além de ter relações altas com a fertilidade de amostras de sêmen bovino congeladas em programas de inseminação artificial (ARRUDA et al., 2010; OLIVEIRA et al., 2013; ZUBAIR et al., 2014).

A falta de padronização do teste hiposmótico em relação à concentração do sêmen incubado com a solução hiposmótica, pode ser um problema na visualização dos espermatozoides da lâmina em microscopia óptica, tanto em sêmen in natura quanto congelado/descongelado. Revell e Mrode (1994) recomendaram a utilização de 5 a $10 \mu \mathrm{L}$ de sêmen in natura (dependendo da concentração do ejaculado) em $1 \mathrm{~mL}$ de solução hiposmótica, mas se o ejaculado estiver com aspecto aquoso ou opalescente a leitura da lâmina em contraste de fase se torna difícil devido a pequena quantidade de espermatozoides por campo. Isto se torna mais problemático quando se trata de amostras congeladas/descongeladas.

Siqueira et al. (2007), Borges et al. (2011) e Oliveira et al. (2012) utilizaram $20 \mu \mathrm{L}$ de sêmen congelado/descongelado em $1 \mathrm{~mL}$ de solução hiposmótica, enquanto Vera-Munoz et al. (2009) utilizaram $100 \mu \mathrm{L}$ de sêmen congelado/descongelado em $1 \mathrm{~mL}$ de solução hiposmótica. Outros autores utilizaram diferentes proporções de sêmen congelado/descongelado e solução hiposmótica, Tuncer et al. (2011) utilizaram $250 \mu \mathrm{L}$ de sêmen em $300 \mu \mathrm{L}$ de solução hiposmótica e Nava-Trujillo et al. (2011) $30 \mu \mathrm{L}$ de sêmen em $300 \mu \mathrm{L}$ de solução hiposmótica.

Observando os diversos protocolos utilizados, este estudo teve como objetivo avaliar as diferentes proporções sêmen: solução hiposmótica na realização do teste hiposmótico e suas relações com a qualidade e congelabilidade do sêmen in natura e congelado/descongelado de touros adultos da raça Nelore.

\section{Material e Métodos}

Foram utilizados neste experimento três touros adultos da raça Nelore com idades variando de quatro a sete anos criados em condições extensivas no Setor de Bovinocultura de Corte da Universidade Federal de Viçosa . Foram obtidos pelo método de eletroejaculação, cinco ejaculados por touro. No sêmen in natura foram feitas as avaliações física e mor- fológica, a coloração supravital e o teste hiposmótico. Posteriormente, cada ejaculado foi diluído em meio diluidor utilizado para a criopreservação ${ }^{8}$ e congelado em palhetas $(0,25$ $\mathrm{mL}$ ), numa concentração de 30 milhões de espermatozoides total por dose e congeladas conforme protocolo preconizado por Morrow (1986).

A avaliação física do sêmen foi feita analisando os seguintes aspectos: volume, turbilhonamento (0-5), motilidade espermática retilínea progressiva $(0-100 \%)$ e o vigor espermático (0-5), conforme parâmetros do Colégio Brasileiro de Reprodução Animal (CBRA, 2013).

Em um tubo contendo $1 \mathrm{~mL}$ de solução formol-salina tamponada foi acondicionada uma alíquota do ejaculado suficiente para turvar a solução, para a análise morfológica dos espermatozoides por meio de preparação úmida e utilização de microscopia de contraste de fase em aumento de 100X (sob uma gota de óleo de imersão). Foram contabilizadas 400 células por ejaculado, e os defeitos espermáticos foram mensurados em percentagem segundo os critérios de classificação adotados por Blom (1973) e preconizados pelo Colégio Brasileiro de Reprodução Animal - CBRA (2013).

Para o teste hiposmótico foi utilizada uma solução de citrato de sódio e frutose com osmolaridade de 100 $\mathrm{mOsm} / \mathrm{Kg}$ com 15 minutos de período de incubação a $37^{\circ} \mathrm{C}$, tanto em sêmen in natura quanto congelado/descongelado (MARTINS et al., 2011a). Foram utilizados quatro volumes de sêmen em $1 \mathrm{~mL}$ de solução hiposmótica: 10, 20, 50 e $100 \mu \mathrm{L}$. As osmolaridades foram ajustadas e aferidas pelo método crioscópico (HENRIQUES; ROSADO, 1999). Depois do período de incubação, foram acrescentados $0,5 \mathrm{~mL}$ de solução de formol-salina tamponada para a fixação dos espermatozoides e posteriormente feita a análise em microscopia de contraste de fase. Foram contabilizados 100 espermatozoides em aumento de $1000 \mathrm{X}$ e obtido o percentual de espermatozoides que tiveram seu flagelo curvando-se junto à membrana expandida, para depois ser calculada a percentagem de espermatozoides reativos subtraindo do percentual de defeitos de cauda registrado no sêmen in natura (MELO; HENRY, 1999). O mesmo procedimento para o sêmen in natura em relação ao teste hiposmótico foi realizado no sêmen congelado/descongelado.

A coloração supravital foi utilizada para a avaliação da integridade física da membrana plasmática, utilizando solução de Eosina (1\%) e Nigrosina (5 \%) conforme descrito

${ }^{8}$ Bioxcell $^{\circ}$, VMI, L Aigle, França 
por Swanson e Bearden (1951). Para o teste de termo-resistência lento foi utilizado um banho-maria com circulador de água a $37{ }^{\circ} \mathrm{C}$ durante três horas. O sêmen congelado em palhetas de $0,25 \mathrm{~mL}$ após seu descongelamento a $37{ }^{\circ} \mathrm{C}$ por 30 segundos foram acondicionados e homogeneizados em tubos plásticos de 1,5 mL. Durante o período de incubação, as amostras de sêmen foram avaliadas quanto aos aspectos de motilidade espermática progressiva retilínea e o vigor espermático nos intervalos de $0,60,120,180$ minutos.

A coloração fluorescente foi realizada utilizando-se dois fluocromos, o diacetato de carboxifluresceína e o iodeto de propídeo, segundo a técnica descrita por Harrison e Vickers (1990). Foram contabilizados 100 espermatozoides e classificados em integros, parciamente lesados e lesados. Os espermatozoides íntegros coram-se pelo diacetato de carboxifluresceína (verdes em toda extensão), os parcialmente lesados, o acrossomo e a membrana coram-se pelo diacetato de carboxifluresceína, mas o núcleo se cora com o iodeto de propídeo (vermelho), e os lesados se coram de vermelho.

Para a análise estatística foi utilizado o software SAEG versão 9.1. Os dados foram arranjados em fatorial de dois tipos de sêmen (in natura e congelado/descongedo), quatro quantidades de sêmen em $1 \mathrm{~mL}(10,20,50$ e $100 \mu \mathrm{L})$ na realização do teste hiposmótico, sendo oito tratamentos por ejaculado. Análises descritivas quanto as médias, desvios-padrão e coeficientes de variação foram feitas para todas as características estudadas. $\mathrm{O}$ teste de Lilliefors foi utilizado para verificação de normalidade das respostas das variáveis estudadas, exceto o vigor espermático que é classificada como uma variável não paramétrica. A homogeneidade das variâncias para cada grupo de tratamento foi estudada, utilizando-se o teste de Cochran-Bartlett. A análise de variância foi utilizada para avaliar o efeito das diferentes concentrações de sêmen na realização do teste hiposmótico. Foram também avaliados os efeitos dos touros e ejaculados nos aspectos físicos e morfológicos, teste hiposmótico, termo-resistência lento, e coloração fluorescente. Quando foi detectado efeito pelo teste $\mathrm{F}$, os dados foram comparados pelo teste $\mathrm{T}$ e o teste de Tukey (5\%). O teste de Kruskal-Wallis (5\%) foi realizado para a análise do efeito dos touros para vigor espermático após realizado o teste de termoresistência no sêmen congelado/descongelado. Correlação simples de Pearson foi realizada para verificar as relações das características seminais entre os testes realizados. Testaram-se as interações entre os tipos de sêmen (congelado/descongelado e in natura) e quatro concentrações de sêmen na realização do teste hiposmótico.

\section{Resultados e Discussão}

Os touros foram classificados como aptos à reprodução segundo os critérios preconizados pelo CBRA (2013), apresentando ejaculados de boa qualidade durante todo período experimental. Ao se analisar os aspectos físicos e morfológicos do sêmen in natura, os animais apenas diferiram entre si em relação aos defeitos espermáticos maiores $(\mathrm{p}<0,05)$. As médias de motilidade espermática progressiva retilínea, vigor espermático, turbilhonamento e concentração espermática foram $77,3 \pm 5,1 ; 3,3 \pm 0,3 ; 1,6 \pm 1,1$ e $682,0 \pm 283,0$; respectivamente. Borges et al. (2011) observaram médias superiores de turbilhonamento, concentração espermática por $\mathrm{mL}$ em milhões e vigor (4,2; 1.468 e 4,4; respectivamente), mas uma média semelhante de motilidade espermática retilínea progressiva $(76,8 \%)$ no sêmen in natura. As médias dos percentuais de defeitos maiores, menores e totais foram $11,5 \pm 4,8 ; 2,4 \pm 1,6 ; 13,9 \pm 5,5$; respectivamente. Brito et al. (2002) e Borges et al. (2011) observaram médias superiores de defeitos espermáticos maiores, menores e totais no sêmen in natura.

Os resultados obtidos no presente estudo (Tabela 1), corroboram com os de Vera-Munoz et al. (2009), Borges et al. (2011) e Martins et al. (2011a) onde o percentual de espermatozoides reativos no teste hiposmótico do sêmen in natura foi maior que o congelado/descongelado $(\mathrm{p}<0,05)$ (Tabela 1), demonstrando os danos causados à membrana plasmática pelo processo de criopreservação. Após o processo de criopreservação, houve uma perda de $50 \%$ da motilidade progressiva retilínea espermática, valor aceitável conforme Watson (2000). Mas se observou uma perda de 52\% do teste supravital e $70 \%$ do teste hiposmótico de células viáveis. Isto porque o processo de criopreservação promove danos às membranas plasmáticas dos espermatozoides, alterando os domínios e função de várias proteínas (proteínas transportadoras de íons, por exemplo) importantes no metabolismo espermático, causando injúrias subletais e letais aos espermatozoides (WATSON, 2000).

Tabela 1: Valores médios e desvios-padrão do teste supravital e percentual de espermatozoides reativos após incubação em solução hiposmótica em sêmen in natura e congelado/descongelado de touros da raça Nelore.

\begin{tabular}{ccccc}
\hline Touros & Supravital $(\mathrm{N})$ & Hipo $(\mathrm{N})$ & Supravital $(\mathrm{C} / \mathrm{D})$ & Hipo $(\mathrm{C} / \mathrm{D})$ \\
\hline 1 & $80,8 \pm 5,5^{\mathrm{a}}$ & $68,3 \pm 16^{\mathrm{a}}$ & $35,4 \pm 12,8^{\mathrm{a}}$ & $19,3 \pm 8,6^{\mathrm{a}}$ \\
2 & $82,6 \pm 8,9^{\mathrm{a}}$ & $70,9 \pm 7,1^{\mathrm{a}}$ & $47,2 \pm 5,4^{\mathrm{a}}$ & $23,3 \pm 5,3^{\mathrm{a}}$ \\
3 & $85,0 \pm 3,2^{\mathrm{a}}$ & $68,8 \pm 13,4^{\mathrm{a}}$ & $37,0 \pm 10,1^{\mathrm{a}}$ & $25,0 \pm 6,9^{\mathrm{a}}$ \\
\hline Média & $82,8 \pm 6,1$ & $69,3 \pm 11,8$ & $39,8 \pm 10,6$ & $20,5 \pm 6,8$ \\
\hline
\end{tabular}

$\overline{\mathrm{a}, \mathrm{b}, \mathrm{c}}=$ letras minúsculas iguais na mesma coluna indicam que não houve diferença $(\mathrm{p}<0,05)$ pela ANOVA a $5 \%$; Supravital $(\mathrm{N})=$ coloração com eosina-nigrosina do sêmen in natura $(\%)$; Hipo $(\mathrm{N})$ = percentual de espermatozoides reativos no sêmen in natura após o teste hiposmótico; Supravital $(\mathrm{C} / \mathrm{D})$ = coloração com eosina-nigrosina do sêmen congelado/descongelado (\%); Hipo (C/D) = percentual de espermatozoides reativos no sêmen congelado/descongelado após o teste hiposmótico.

Os aspectos físicos (motilidade espermática progressiva retilínea e vigor espermático) pós-descongelamento não diferiram entre os ejaculados obtidos dos diferentes touros ( $\mathrm{p}>0,05)$ (Tabela 2). As médias da motilidade espermá- tica progressiva retilínea do sêmen congelado/descongelado e após a realização do teste de termoresistência lento foram mais baixas que as observadas por outros autores em touros adultos da raça Nelore (SIQUEIRA et al., 2007; BORGES et 
al., 2011).

Neste estudo $60 \%$ das partidas de sêmen congeladas foram classificadas como aptas para uso em programas de inseminação artificial, segundo os critérios preconizados pelo CBRA (2013). O animal dois apresentou os melhores congelamentos, com $100 \%$ das partidas de sêmen congelado aptas para uso em programas de inseminação artificial, enquanto que os outros dois animais apenas com $40 \%$ de suas partidas aprovadas, porém não foram observadas diferenças das médias dos touros na motilidade espermática progressiva retilínea e vigor espermático após a realização do teste de termo-resistência $(p>0,05)$. Foram observadas diferenças entre as médias dos touros na diminuição da motilidade espermática progressiva retilínea e vigor espermático após a realização do teste de termo-resistência $(\mathrm{p}<0,05)$. $\mathrm{O}$ touro dois teve a menor perda da motilidade progressiva retilínea e vigor espermáticos durante o teste de termo-resistência $(\mathrm{p}<0,05)$ (Tabela 2), sendo o teste de termo-resistência o único teste complementar que mostrou diferenças entre as médias dos touros de alta (touro 2), média e/ou baixa (touros 1 e 3 ) congelabilidade do sêmen.

Vianna et al. (2009) utilizando em um programa de inseminação artificial amostras de sêmen congelado/descongelado reprovadas pelo teste de termo-resistência lento $(0 \%$ de motilidade espermática progressiva retilínea após o término do teste), observou taxas de gestação semelhantes às amostras congeladas/descongeladas que apresentaram acima de $40 \%$ de motilidade progressiva retilínea espermática após a realização do teste. Segundo estes autores, o teste de termo-resistência lento é capaz de avaliar touros e ejaculados com boa congelabilidade, mas não consegue predizer a capacidade fecundante em programas de inseminação artificial.

Tabela 2: Valores médios e desvios-padrão dos aspectos físicos do sêmen de touros adultos da raça Nelore congelado/descongelado e após 3 horas de incubação.

\begin{tabular}{ccccccc}
\hline Touros & Motilidade & Vigor & Motilidade $3^{\mathrm{a}}$ hora & $\downarrow$ Motilidade & Vigor $3^{\mathrm{a}}$ hora & $\downarrow$ Vigor \\
\hline 1 & $38,0 \pm 5,7^{\mathrm{a}}$ & $3,1 \pm 0,2^{\mathrm{A}}$ & $13,0 \pm 7,5^{\mathrm{a}}$ & $25,0 \pm 5^{\mathrm{a}}$ & $2,4 \pm 0,5^{\mathrm{A}}$ & $0,7 \pm 0,6^{\mathrm{AB}}$ \\
2 & $39,0 \pm 6,5^{\mathrm{a}}$ & $3,4 \pm 0,2^{\mathrm{A}}$ & $29,0 \pm 7,4^{\mathrm{a}}$ & $10,0 \pm 6,1^{\mathrm{b}}$ & $3,1 \pm 0,2^{\mathrm{A}}$ & $0,3 \pm 0,2^{\mathrm{B}}$ \\
3 & $40,0 \pm 11,7^{\mathrm{a}}$ & $3,6 \pm 0,4^{\mathrm{A}}$ & $17,0 \pm 23,8^{\mathrm{a}}$ & $23,0 \pm 12,5^{\mathrm{ab}}$ & $1,3 \pm 1,7^{\mathrm{A}}$ & $2,3 \pm 1,4^{\mathrm{A}}$ \\
\hline Média & $39,0 \pm 7,8$ & $3,3 \pm 0,3$ & $19,6 \pm 15,6$ & $19,3 \pm 10,4$ & $2,2 \pm 1,2$ & $1,1 \pm 1,2$ \\
\hline
\end{tabular}

$\overline{\mathrm{a}, \mathrm{b}, \mathrm{c}}=$ letras minúsculas diferentes na mesma coluna indicam diferença $(\mathrm{p}<0,05)$ pelo teste de Tuckey a $5 \%$. $\mathrm{A}, \mathrm{B}, \mathrm{C}=$ letras maiúsculas diferentes na mesma coluna indicam diferença $(\mathrm{p}<0,05)$ pelo teste de Kruskal-Wallis a 5\%. Motilidade $=$ motilidade espermática $(\%)$; Vigor $=$ vigor espermático (0-5); Motilidade $3^{\mathrm{a}}$ hora $=$ motilidade espermática após 3 horas de incubação (\%); $\downarrow$ Motilidade $=$ diminuição da motilidade espermática após 3 horas de incubação; Vigor $3^{\mathrm{a}}$ hora $=$ vigor espermático após 3 horas de incubação $(0-5) ; \downarrow$ Vigor $3^{\mathrm{a}}$ hora $=$ diminuição do vigor espermático após 3 horas de incubação.

A média do percentual de células espermáticas viáveis registradas no teste supravital $(39,8 \%)$ (Tabela 3$)$ foi menor do que o verificado por Martins et al. (2011a) (51,4\%) e Tanghe et al. (2002) (69,5\%) com touros taurinos. Zúccari et al. (2009) utilizando touros adultos da raça Nelore observaram um percentual de $51,6 \%$ de espermatozoides viáveis no teste supravital. Tanghe et al. (2002) obtiveram uma média maior que a deste experimento $(47,1 \%)$ em relação à motilidade espermática progressiva retilínea após o descongelamento (39\%) (Tabela 2). No presente estudo, não foram observadas diferenças nos percentuais de células íntegras nos testes supravital e hiposmótico entre os ejaculados dos diferentes touros, no sêmen in natura e no congelado/descongelado ( $\mathrm{p}>0,05)$ (Tabela 2).

A média de espermatozoides reativos no teste hiposmótico foi inferior a Siqueira et al. (2007) (37,9\%), que utilizaram sêmen congelado/descongelado de touros adultos da raça Nelore. Bacinoglu et al. (2008) e Mocé e Graham (2008) também observaram médias superiores ao presente experimento (Tabela 3), porém utilizando touros taurinos. Zúccari et al. (2009) observaram uma média semelhante ao presente experimento.

A média dos percentuais dos espermatozoides íntegros avaliados sob microscopia de epifluorescência foi muito baixa em relação a outros autores (BRITO et al., 2003; BORGES et al., 2011, MARTINS et al., 2011a). Corroborando com Brito et al. (2003) e Borges et al. (2011) foram observados valores inferiores de espermatozoides viáveis na coloração fluorescente em relação aos testes supravital e hiposmótico em sêmen congelado/descongelado. Não houve diferenças entre as médias dos percentuais de espermatozoides íntegros na coloração fluorescente dos touros $(\mathrm{p}>0,05)$ (Tabela 3).

Tabela 3: Valores médios e desvios-padrão dos resultados dos testes supravital, hipósmótico e coloração fluorescente em sêmen congelado/descongelado de touros adultos da raça Nelore.

\begin{tabular}{cccccc}
\hline Touros & Supravital (C/D) & Hipo $(\mathrm{C} / \mathrm{D})$ & \multicolumn{3}{c}{ Epifluorescência } \\
\cline { 3 - 5 } & & & Intacto & Parcialmente lesado & Lesado \\
\hline 1 & $35,4 \pm 12,8^{\mathrm{a}}$ & $19,3 \pm 8,6^{\mathrm{a}}$ & $9,4 \pm 4,5^{\mathrm{a}}$ & $13,6 \pm 5,7^{\mathrm{a}}$ & $75,0 \pm 8,7^{\mathrm{a}}$ \\
2 & $47,2 \pm 5,4^{\mathrm{a}}$ & $23,3 \pm 5,3^{\mathrm{a}}$ & $11,6 \pm 5,9^{\mathrm{a}}$ & $19,0 \pm 8,9^{\mathrm{a}}$ & $69,4 \pm 11,3^{\mathrm{a}}$ \\
3 & $37,0 \pm 10,1^{\mathrm{a}}$ & $25,0 \pm 6,9^{\mathrm{a}}$ & $10,0 \pm 5,5^{\mathrm{a}}$ & $11,2 \pm 7,3^{\mathrm{a}}$ & $76,8 \pm 10,3^{\mathrm{a}}$ \\
\hline Média & $39,8 \pm 10,6$ & $20,5 \pm 6,8$ & $10,3 \pm 5$ & $14,6 \pm 7,6$ & $73,7 \pm 9,9$ \\
\hline
\end{tabular}

$\overline{a, b, c}=$ letras minúsculas diferentes na mesma coluna indicam diferença $(\mathrm{p}<0,05)$ pelo teste de Tukey a $5 \%$. Supravital $(\mathrm{C} / \mathrm{D})=$ coloração com eosina-nigrosina $(\%)$; Hipo $(\mathrm{C} / \mathrm{D})=$ percentual de espermatozoides reativos após o teste hiposmótico. 
Na tabela 4 observam-se as diferenças entre as médias das concentrações de sêmen in natura na realização do teste hiposmótico $(\mathrm{p}<0,05)$, onde apenas a utilização de $10 \mu \mathrm{L}$ foi diferente $(\mathrm{p}<0,05)$ em relação aos demais volumes utilizados. Pode ser a influência de uma diluição excessiva dos componentes do plasma seminal na preservação da integridade funcional da membrana plasmática durante a incubação hiposmótica (MAXWELL; JOHNSON, 1999). Isto não foi observado no sêmen congelado/descongelado, pois não houve diferenças entre as proporções de sêmen: solução hiposmótica na realização do teste hiposmótico em sêmen congelado/descongelado $(\mathrm{p}>0,05)$.

Tabela 4: Valores médios e desvios-padrão do percentual de espermatozoides reativos em sêmen in natura e congelado/ descongelado de touros adultos da raça Nelore, após incubação em solução hipoosmótica com diferentes volumes de sêmen.

\begin{tabular}{ccc}
\hline Amostra de sêmen & Hipo $(\mathrm{N})$ & Hipo $(\mathrm{C} / \mathrm{D})$ \\
\hline $10 \mu \mathrm{L}$ & $69,3 \pm 11,8^{\mathrm{b}}$ & $22,5 \pm 7,0^{\mathrm{a}}$ \\
$20 \mu \mathrm{L}$ & $76,6 \pm 8,1^{\mathrm{ab}}$ & $21,4 \pm 7,4^{\mathrm{a}}$ \\
$50 \mu \mathrm{L}$ & $82,2 \pm 3,6^{\mathrm{a}}$ & $20,4 \pm 7,3^{\mathrm{a}}$ \\
$100 \mu \mathrm{L}$ & $78,4 \pm 6,6^{\mathrm{a}}$ & $17,8 \pm 5,1^{\mathrm{a}}$ \\
\hline Média & $69,3 \pm 11,8$ & $20,5 \pm 6,8$ \\
\hline a,b,c $=$ letras minúsculas diferentes na mesma coluna indicam dife- \\
rença (p<0,05) pelo teste de Tukey a 5\%; Hipo (N) = percentual de \\
espermatozoides reativos no sêmen in natura após o teste hipos- \\
mótico; Hipo (C/D) = percentual de espermatozoides reativos no \\
sêmen congelado/descongelado após o teste hipoosmótico.
\end{tabular}

Nenhuma correlação foi observada do teste hiposmótico com os aspectos físicos, morfológicos e os testes complementares realizado em sêmen in natura e congelado/ descongelado, discordando dos achados de Zúccari et al. (2009) que observaram correlações altas do teste hiposmótico os outros teste complementares.

Correlações médias e positivas foram observadas entre o teste supravital em sêmen congelado/descongelado e vigor espermático após o descongelamento $(0,47)$ e a motilidade espermática progressiva retilínea após a realização do teste de termo-resistência $(0,48)$, como também uma correlação média e negativa com a diminuição da motilidade espermática progressiva retilínea durante o teste de termo-resistência $(-0,44)$, que demonstra a importância do supravital em predizer a congelabilidade do sêmen congelado/descongelado. Sharma et al. (2012) observaram correlações altas da motilidade espermática progressiva retilínea com o teste supravital $(0,74)$ e com o teste hiposmótico $(0,73)$ em sêmen congelado/descongelado de touros mestiços. Correlações positivas e médias também foram observadas do percentual de espermatozoides íntegros após a coloração fluorescente realizada em sêmen congelado/descongelado com a motilidade espermática progressiva retilínea após o descongelamento $(0,50)$ e após a realização do teste de termo-resistência $(0,52)$.

Nenhum teste de integridade de membrana plasmática dos espermatozoides foi capaz de classificar os touros quanto à congelabilidade do sêmen. $\mathrm{O}$ teste hiposmótico não pode ser usado para predizer o status reprodutivo de touros adultos da raça Nelore em sistema extensivo de criação (MARTINS et al., 2011b), bem como classificar os touros quanto à congelabilidade do sêmen (Martins et al., 2011a).

A coloração fluorescente pode ser uma importante ferramenta complementar para avaliação de sêmen bovino congelado/descongelado, haja vista a relação observada neste estudo com as principais características de qualidade seminal e testes complementares já utilizados como rotina na avaliação da qualidade seminal (teste de termo-resistência e supravital). Com os resultados deste experimento pode-se concluir que o teste hiposmótico pode ser realizado com 20 a $100 \mu \mathrm{L}$ em sêmen in natura, e 10 a $100 \mu \mathrm{L}$ de sêmen congelado/descongelado em $1 \mathrm{~mL}$ de solução hiposmótica, sem interferir em seus resultados, mas deve-se optar por $100 \mu \mathrm{L}$ tanto para sêmen in natura e congelado/descongelado, porque melhora consideravelmente a leitura das lâminas.

\section{Agradecimentos}

Os autores deste trabalho agradecem ao órgão financiador (CAPES) pela concessão de bolsa PROSUP.

\section{Referências}

ARRUDA, R. L. et al. Técnicas para avaliação laboratorial da integridade estrutural e funcional do sêmen congelado de touros. Revista Brasileira de Reprodução Animal, v. 34, n. 3, p. 168-184, 2010.

BACINOGLU, S. et al. The potential fertility estimation capacity of the hypoosmotic swelling test, the thermal stress test and a modified cervical mucus penetration test in the bovine. Animal Reproduction Science, v. 104, p. 38-46, 2008.

BLOM, E. The ultrastructure of some characteristic sperm deffects and a proposal for a new classification of the Bull spermiogram. Nord Veterinary Médicine, v. 25, p. 383339, 1973.

BORGES, J. C. et al. Membrana plasmática de espermatozoides bovinos: efeito de metabólitos do oxigênio, antioxidantes e criopreservação. Revista Brasileira de Reprodução Animal, v. 35, p. 303-314, 2011.

BRITO, L. F. C. et al. Effect of age and genetic group on characteristics of the scrotum, testes and testicular vascular cones, and on sperm production and semen quality in AI bulls in Brazil. Theriogenology, v. 58, p. 1175-1186, 2002.

BRITO, L. F. C. et al. Comparison of methods to evaluate the plasmalemma of bovine sperm and their relationship with in vitro fertilization rate. Theriogenology, v. 60, p. 1539-1551, 2003.

\section{CBRA - COLÉGIO BRASILEIRO DE REPRODUÇÃO} ANIMAL. Manual para exame andrológico e avaliação de sêmen animal. 3. ed. Belo Horizonte, 2013. 104 p.

HARRISON, R. A.; VICKERS, S. E. Use of fluorescent probes to assess membrane integrity in mammalian spermatozoa. Journal of Reproduction and Fertility, v. 88 , p. 343-352, 1990. 
HENRIQUES, G. S.; ROSADO, G. P. Formulação de dietas enterais artesanais e determinação da osmolaridade pelo método crioscópico. Revista de Nutrição de Campinas, v. 12, p. 225-232, 1999.

MARTINS, L. F. et al. Avaliação de diferentes osmolaridades de soluções hiposmóticas e tempos de incubação no teste hiposmótico do sêmen de touros Nelore. Revista Brasileira de Zootecnia, v. 40, n. 7, p. 1519-1525, 2011a.

MARTINS, L. F. et al. Correlation between hypoosmotic swelling test and breeding soundness evaluation of adult Nelore bulls. Italian Journal of Animal Science, v. 41, p. 217-219, 2011b.

MAXWELL, W. M. C.; JOHNSON, L. A. Physiology of spermatozoa at high dilution rates: The influence of seminal plasma. Theriogenology, v. 52, p. 1353-1362, 1999.

MELO, M. I. V.; HENRY, M. Teste hiposmótico na avaliação de sêmen eqüino. Arquivo Brasileiro de Veterinária e Zootecnia, v. 51, n. 1, p.71-78, 1999.

MOCÉ, E.; GRAHAM, J. K. In vitro evaluation of sperm quality. Animal Reproduction Science, v. 105, p. 104-118, 2008.

MORROW, D. A. Current therapy in theriogenology. Philadelphia: W. B. Saunders, 1986, p. 1143.

NAVA-TRUJILLO, H. et al. Use of water test to assess the sperm membrane functional integrity in cryopreserved bull semen. Revista Científica, v. 21, p. 211-214, 2011.

OLIVEIRA, L. Z. et al. Assessment of in vitro sperm charcteristics and their importance in the predicition of conception rate in a bovine timed-AI program. Animal Reproduction Science, v. 137, p. 145-155, 2013.

OLIVEIRA, L. Z. et al. Assessment of field fertility and several in vitro sperm characteristics following the use of different Angus sires in a timed-AI program with suckled Nelore cows. Livestock Science, v. 146, p. 38-46, 2012.

REVELL, S. G., MRODE, R. A. An osmotic resistance test for bovine semen. Animal Reproduction Science, v. 36, p. 77-86, 1994.

SHARMA, M. et al. Inter relationship between some routine semen evaluation parameters in Jersey $\mathrm{x}$ local hil cattel crossbred bulls. Open Veterinary Journal, v. 2, p. 26-31, 2012.

SIQUEIRA, J. B. et al. Relação da taxa de gestação com sêmen bovino congelado e testes de avaliação espermática in vitro. Revista Brasileira de Zootecnia, v. 36, p. $387-$ 395, 2007.

SWANSON, E. W.; BEARDEN, H. J. An eosin-nigrosin stain for differentiating live and dead bovine spermatozoa.
Journal of Animal Science, v. 10, p. 981-987, 1951.

TANGHE, S. et al. Assessment of different sperm quality parameters to predict in vitro fertility of bulls. Reproduction in Domestic Animals, v. 37, v. 127-137, 2002.

TUNCER, P. B. et al. Effect of glutamine and sugars after bull spermatozoa cryopreservation. Theriogenology, v. 75, p. 1459-1465, 2011.

VERA-MUNOZ, O. et al. Effect of semen dilution to low-sperm number per dose on motility and functionality of cryopreserved bovine spermatozoa using low-density lipoproteins (LDL) extender : Comparison to Triladyl ${ }^{\circledR}$ and Bioxcell®. Theriogenology, v. 71, p. 895-900, 2009.

VIANNA, F. P. et al. Thermoresistance sperm tests are not predictive of potential fertility for cryopreserved bull semen. Animal Reproduction Science, v. 113, p. 279-282, 2009.

WATSON, P. F. The causes of fertility with cryopreserved semen. Animal Reproduction Science, v. 61, p. 481-492, 2000 .

ZUBAIR, M.; AHMAD, M.; JAMIL, H. Review on the screening of semen by hypo-osmotic swelling test. Andrologia, v. 15, p. 1-7, 2014.

ZÚCCARI, C. E. S. N. et al. Correlação entre métodos de avaliação da integridade da membrana plasmática do espermatozoide bovino criopreservado. Revista Brasileira de Saúde e Produção Animal, v. 10, p. 678-684, 2009.

Recebido em: 11.12.2014 Aceito em: 31.03.2015 